\title{
Display dimming model characterized by three-dimensional ergonomic study
}

\author{
Dandan Hou $\odot,{ }^{\text {a }}$ Wei Xu, ${ }^{\text {a }}$ Shuo Jing, ${ }^{\text {a,b }}$ and Yandan Lin $\odot^{\text {a,c, } *}$ \\ ${ }^{a}$ Fudan University, Institute for Electric Light Sources, Shanghai, China \\ ${ }^{b}$ Fudan University, Institute for Six-Sector Economy, Shanghai, China \\ ${ }^{c}$ Fudan University, Institute for Engineering and Applied Technology, Shanghai, China
}

\begin{abstract}
Self-luminous display devices are essential in various working environments, such as aircraft cockpits and the driver cab of vehicles and trains, where the external light environment varies drastically. Owing to the significant illumination changes, auto-adjusted display luminance based on ambient lighting is necessary for drivers to work efficiently and comfortably. This study proposes a display dimming model based on three dimensions of ergonomic testing, consisting of visual performance (VP), visual comfort (VC), and visual fatigue (VF). Five ambient illuminances, each combined with five display luminance levels, form a total of 25 conditions demonstrated in this experiment. Using a within-subject design, ten observers experienced all the combination conditions. The experiment employs the Anfimov table to test the VP, VC scale to evaluate VC, and VF scale to assess VF. Based on the experimental results, sub-models are constructed to clarify the characteristics of each dimension (VP, VC, and VF). Subsequently, the analytic hierarchy process is employed to construct an evaluation system by calculating the weight of each dimension in the total score. Finally, exponential fitting is utilized to build a widerange display dimming model, which explicitly describes the inherent connection of complex light environment matching. () The Authors. Published by SPIE under a Creative Commons Attribution 4.0 Unported License. Distribution or reproduction of this work in whole or in part requires full attribution of the original publication, including its DOI. [DOI: 10.1117/1.OE.60.3.035110]
\end{abstract}

Keywords: adaptive dimming; display; visual performance; visual fatigue; visual comfort.

Paper 20201408 received Dec. 7, 2020; accepted for publication Mar. 8, 2021; published online Mar. 30, 2021.

\section{Introduction}

Self-luminous display devices, such as computers, mobile phones, and tablets, have become indispensable in our daily lives. Moreover, display devices are increasingly replacing traditional mechanical buttons, meters, and light guide panels in many essential scenarios of humancomputer interactions, such as aircraft cockpits and the driver cab of vehicles and trains. However, with the convenience and diverse interfaces enabled by the widespread use of displays, some challenges still exist. To enable efficient use when there are changes in the external light environment, the display must be equipped with an adaptive luminance adjustment algorithm, which matches the light environment and maintains the readability of information. This is important for visual performance (VP) and the effectiveness of human-computer interaction.

Recently, some studies have explored the impact of ambient light, and display luminance on users from different perspectives and have made some progress in both VP and the combined effects of visual comfort (VC) and visual fatigue (VF). In general, the visual capability increased as the display luminance increased. ${ }^{1} \mathrm{Xu}$ and $\mathrm{Zhu}$ suggested that high performance occurs at high display luminance, in contrast to low luminance. ${ }^{2} \mathrm{Lin}^{3}$ also indicated that VP increased with increasing screen luminance. Regarding ambient light, Shen et al. ${ }^{4}$ presented a study on electronic paper displays that showed that the search speed increased as illumination increased from $300 \mathrm{~lx}(45.6 \mathrm{~s}), 700 \mathrm{~lx}(44.18 \mathrm{~s})$ to $1500 \mathrm{~lx}$ (43.24 s) for a fixed display luminance. This indicated that the VP improved with an increase in the ambient illuminance. A similar conclusion appears in Lin's ${ }^{5}$ study, and the results showed that reading comprehension performance was better at $500 \mathrm{~lx}$ and $800 \mathrm{~lx}$ lighting than at $200 \mathrm{~lx}$. In addition to their impact on VP, ambient light and

*Address all correspondence to Yandan Lin, ydlin@fudan.edu.cn 
display luminance also impact VC and VF. Lee and Whang ${ }^{6}$ presented a study in 2012 that showed that the viewer felt intense VF at high luminance levels over $400 \mathrm{~cd} / \mathrm{m}^{2}$. Wang et al. ${ }^{7}$ suggested that luminance is the critical factor that causes VF, and ambient light has a significant influence on subjective comfort evaluation. It was also found that subjective visual discomfort only represented a part of mental workload, not the whole VF rating. ${ }^{7}$ Therefore, it is important to consider both VC and VF in two dimensions. Base on the above analysis, the definition of the optimal display luminance requires comprehensive consideration of the VP, VF, and $\mathrm{VC}$ dimensions. However, previous studies typically considered one or two of these three dimensions (3D), and an optimal display luminance model that integrates all 3D needs to be explored in depth.

In addition to the independent influence of ambient illuminance or display luminance, the mismatch between ambient light and display luminance is a more important issue in practical applications. Yang et al. explored the transient effects of extreme weather on pilots' visual behavior during flight and demonstrated that pulse-changed and step-changed lighting can result in the temporary visual impairment of pilots. ${ }^{89}$ Rodriguez and Pattini ${ }^{10}$ investigated the influence of ambient lighting with a large glare light source on the display VP. The results showed that memory ability decreased significantly as the glare intensity increased. ${ }^{11,12}$

Hence, some recent studies have also focused on the interaction between multiple factors (ambient illuminance and display luminance). These studies explored the optimal display luminance or ambient illuminance under particular conditions or ranges. Gallimore and Stouffer ${ }^{13}$ showed that background luminance and surrounding luminance revealed significant interactive effects on VP. Lin and Huang ${ }^{14}$ suggested that the combination of ambient illumination and screen luminance are the key factors for users' VP. Additionally, research by radiologists showed that the correct LED backlight luminance during different light conditions may positively affect the perception of the examined sample and reduce VF. ${ }^{15} \mathrm{Na}$ and Suk explored the optimal luminance of a smartphone under low illuminance. They obtained the optimal luminance for initial viewing $\left(10 \mathrm{~cd} / \mathrm{m}^{2}\right)$ and continuous viewing $\left(40 \mathrm{~cd} / \mathrm{m}^{2}\right)$ based on users' subjective judgments. They also proposed an adaptive display model, in which display luminance increases with the watching time. ${ }^{16}$ De Moor et al. investigated how readability and VP varied with seven different combinations of ambient luminance (ranging from 256 to $1536 \mathrm{~cd} / \mathrm{m}^{2}$ ) and display luminance (ranging from 300 to $680 \mathrm{~cd} / \mathrm{m}^{2}$ ). The results showed that with a decrease in contrast and increase in ambient luminance, the self-reported valence decreased, and the annoyance and VF increased. ${ }^{1,17}$ However, the ambient illuminance and display luminance involved in these studies are within a specific small range. In addition, most of them are based only on VP, without consideration of the effects of comfort and VF. Therefore, a comprehensive wide-range display dimming model is necessary.

Thus, this study aims to explore a display-dimming model that is suitable for a working environment. The optimization principle of this model is to enhance VP and VC and simultaneously reduce VF. Based on this, five ambient lighting levels, each with five display luminance levels used as the model input, are investigated in this study. The sub-models of each dimension are carefully investigated in advance. A wide-range dimming curve model is then developed to provide a matching algorithm for the ambient illumination and display luminance.

\section{Methods}

\subsection{Experimental Setup}

A full-scale driving simulator in a simulated cockpit was used in this study for the participants to be immersed in the pilot's perspective while observing the display. Participants were asked to sit in the driver's seat and fasten their seat belts to ensure that the horizontal distance between the eye position and display was $900 \mathrm{~mm}$. The eye position height of each subject was maintained at $1200 \mathrm{~mm}$ by adjusting the seat height.

The display is embedded in the analog operation panel at an angle of $120 \mathrm{deg}$ to the horizontal plane. Six high-power LED floodlights outside the left-wing porthole of the aircraft were used to produce five ambient lighting conditions. An extra metal halide lamp in the lower right 


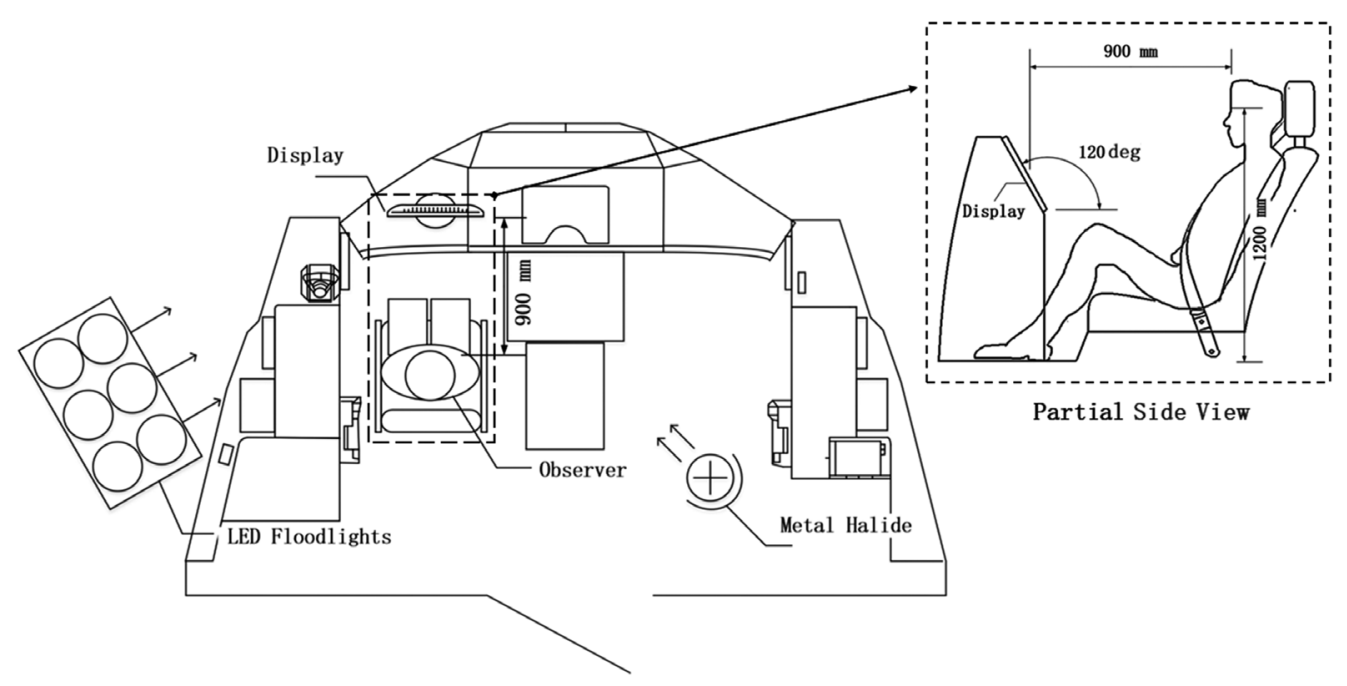

Fig. 1 Layout of the simulated cockpit used in the experiment.

corner of the cockpit was used to achieve the two highest illumination conditions and enhance the display surface uniformity. No direct glare or secondary reflection glare is caused by these lamps in the view of the observers. The layout of the simulated cockpit is shown in Fig. 1.

\subsection{Lighting Conditions}

According to the Weber-Fechner law, human psychological perception is proportional to the logarithm of physical stimulation. Hence, the ambient illumination and display luminance values adopted in this study are uniformly distributed on a logarithmic scale over a wide range. The ambient illumination was set to $1,10,100,1000$, and $2700 \mathrm{~lx}$. The use of $2700 \mathrm{l}$ 'th instead of 10,000 l'th is owing to the reduction of the ambient illumination by the light shield of the display, the driver's sunglasses in a driving cockpit, and the limitations of the laboratory equipment. The display luminance is set to $1,10,100,1000$, and 316 (equals to $10^{2.5}$ ) $\mathrm{cd} / \mathrm{m}^{2}$. The value of $316 \mathrm{~cd} / \mathrm{m}^{2}$ served as an interpolation condition to improve the precision of the data structure.

A detailed physical parameter list is given in Table 1. The ambient illumination and optotype luminance are defined as variables, and the display contrast, line width, color of the optotypes, and color temperature of the ambient light remain constant.

Some previous studies have shown that reading performance is better for dark text against a light background (positive polarity) than for light text against a dark background (negative polarity). ${ }^{18-20}$ However, another study showed that display polarity does not affect readability. ${ }^{21}$ The proofreading speed and accuracy were unaffected by the display polarity. Furthermore, participants' preferences showed no significant differences in display polarity. ${ }^{22}$ Therefore,

Table 1 Physical parameters for experimental lighting conditions.

\begin{tabular}{lcc}
\hline \hline Parameter type & Parameter name & Values \\
\hline Variables & Ambient illuminance $(\mathrm{Ix})$ & $1,10,100,1000,2700$ \\
& Optotype luminance $\left(\mathrm{cd} / \mathrm{m}^{2}\right)$ & $1,10,100,316,1000$ \\
Constant & Contrast ratio & $80: 1$ \\
& Optotypes line width $(\mathrm{mrad})$ & 0.6 \\
& Color temperature $(\mathrm{K})$ & 6500 \\
& $D_{\mathrm{uv}}$ & -0.0089 \\
\hline \hline
\end{tabular}


the display is set to negative polarity in this experiment, allowing improved fidelity to the aircraft cockpit or driver's cab display.

The ambient illuminance is defined as the mean of the illuminance measured at the four corners and center position on the display's surface. ${ }^{23}$ Illuminance uniformity of the display surface in each condition is obtained by dividing the minimum value of the 5 test points by the average value, which is $>0.85$ in each condition. The display luminance is defined as the optotype luminance in a visual task. The contrast ratio is calculated by dividing the optotype luminance by the background luminance of the display. In addition, the optotype line width is calculated using the geometric relationship between the optotype line width on the display and the eye position. The color temperature refers to a cloudless sky, which is equivalent to $6500 \mathrm{~K}$. ${ }^{24}$

The illuminance and optotype luminance are measured using an illuminance meter (CL-200A, Konica Minolta, Japan) and luminance meter (CS-200, Konica Minolta, Japan), respectively.

\subsection{Experimental Process}

Ten subjects participated in this study are undergraduates or graduate students in Fudan University; participants comprised of equal numbers of male and female subjects (average age, 24.20; range, 22 to 26 years). A within-subject design is employed in this experiment, and subjects are asked to complete all conditions in a random order. The sample size is estimated from the results of the pilot study, including the standard deviation and the difference between groups. The confidence level is $95 \%$, and the power of the test is $80 \%$. In addition, about 25 sessions are conducted in five replications to avoid the VF physically caused by the long-time experiment while providing enough time for the participants to fill in the rating scales. ${ }^{4,17,25}$ Participants have normal orthoptists and no other eye diseases. The Ethics Committee of the School of Life Science, Fudan University, approved all procedures (No. BE2042). All participants provided written informed consent before the experiment.

Before the start of the experiment, training on measurement exercises and questionnaire explanations are conducted to enhance the stability of the experiment results. Subsequently, the experimental sessions are executed. Figure 2 shows the timetable for each experimental session, which lasts $15 \mathrm{~min}$. Its description and duration are summarized as follows:

a. dark adaptation (3 $\mathrm{min})$;

b. light adaptation (3 $\mathrm{min})$;

c. visual searching task (5 $\mathrm{min})$;

d. VF scale (2 $\mathrm{min})$;

e. VC scale (2 min);

For each session, dark adaptation is arranged to avoid successive contrast effects and $\mathrm{VF}^{26}$ The 3 min-light adaptation is to make the retina and pupil adapt to the next light condition and in a stable response state. ${ }^{27-29}$

Each participant is required to participate in all 25 conditions. In total, $62.5 \mathrm{~h}$ were spent in experimental sessions: 25 lighting conditions $\times 10$ participants $\times 15$ min per session.

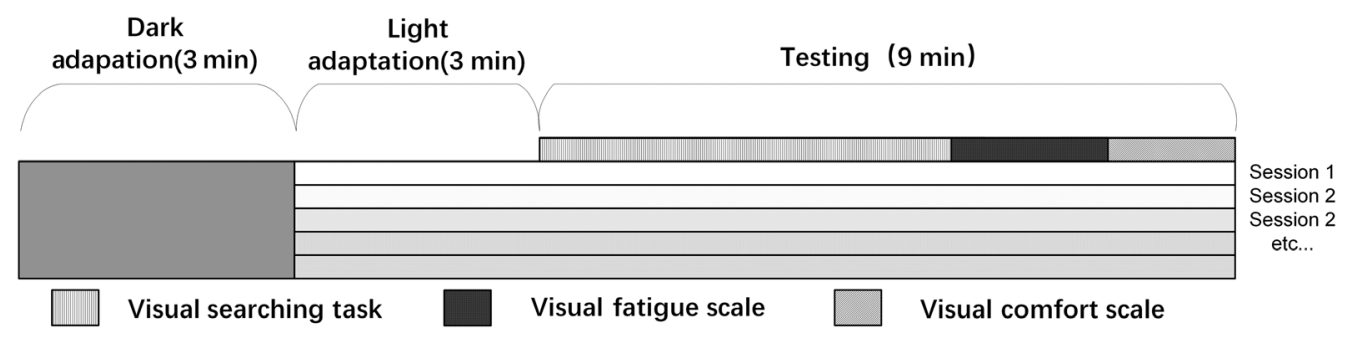

Fig. 2 Test procedure for the experiment: the column bar rectangle represents the visual searching task period; the darker grey gridding rectangle represents the period when the visual fatigue scale is conducted; the diagonal striped rectangles represent the periods when the visual comfort scale is collected. 


\subsection{Measurements}

\subsubsection{Visual performance}

VP means the quality of performance of the visual system of an observer related to central and peripheral vision (CIE S 017:2011). ${ }^{30}$ The Anfimov table is a classical method used to investigate visual searching performance. ${ }^{31-34}$ A graphical user interface (GUI) is programmed (MATLAB, Mathworks) using an automatically generated Anfimov table, which consists of eight letters (A, B, C, B, E, H, K, N, and X). There are a total of 1200 letters in this task, and each letter randomly appears 150 times, as shown in Fig. 3. The participants are asked to cancel the " $\mathrm{B}$ " behind " $\mathrm{H}$ " by clicking it. The program records the hit rate, false-alarm rate, and duration of the task at the end of each test. In addition, the index of mental capacity (IMC) ${ }^{35}$ is employed to represent visual capability, as shown in Eq. (1):

$$
I M C=M / T \times(n-a) / N,
$$

where $T$ denotes the time cost, $M$ represents the total letter count, $N$ represents the number of letters that should be deleted, $n$ denotes the number of letters deleted correctly, and $a$ denotes the number of letters wrongly deleted and those that were missed. Finally, the IMC is calculated as the VP score.

X K B K X B A K A K N C H X A K C B H K B A H A B N C A B X X A C A C H H K H H HXCHKEXKNNXNEXEHEKNECAEBAEAHAKXEEEKCKHKN $K B C C B N H B B K B K E C B E K X E C K X C X K H B C X N N C C B A N E X C E$ KNACNBXABBKEXKXHHHECEHANABACHANKKNKXKBEH EXHBXCECBHXKHACEEABXNCNKHKXHECHAHXEKNACX CEN NXXHNKBXXXBNAXCAECCKXEXABKNENKAXEHXNX A B XEHNAHBXABEACCXBNEBBEXBHAKNBBNEKCHKB B H E NKCHBXECABB EEXHKBNEKBBXKEAACAKHEKEKCBXX $K E E N B X K E N C K K N B B K B A C X B C N B C N B A K E N C E C A B N K C A$ C A C B H A A N H H A N K N X H X A N C N X A A A C B X C A A N XEE CE N HXKNHEKKXEANEEAXENEXNNNHHXKAXCNKECECKN NC $B K X N B E E K A N E E A X K A X C N A E E E A B A H X K H A A X A A H N X K B$ C A X NEEABEHNAAKHKCNNCAEXCXHBNBCEANAKXCXBN $K \times A C H A B E H B X A N H E H K N K N B B N \times K K B E E A C B K N H E E E K C$ X A E EBEBHHKACKHBEHHXCCXANNHABKHBENKCAHN B B NAB NHHB EN B H N A N C B EXHNKBANECEXNKKN N N XE B X A $B \times C E A X K E B K N B H A B E K C A E H N N K N C A E H C C N N H N X A K X X$ EKX HC B NCCKXNKNEKAAKACKE NKHHKKAXEN NCCKKHK $X E B K H C H N A B N K A X A X B C E H C X E B K X E E K B E A X A H C A A K N$ CHAXCCHEXBXABABAAHAHEXXAKKCXXKKCAEENEHXX $X N H H H C K K N B K N N N E N X E X K X C X C C X K X B C A K E B B H A X B K$ HACKCBXENBAXBNNHNKECXHNAHANXEKHNEEHHXCNX $B K E H B A K K B B H E X X B X A H N E H K H E N H C C C A H C C X X K H B N C$ $N K N \times X E H X C X B N X B K A E N A K K E X E A A H C K K N E A C A A X N N X$ KE N N KB X HBA NBAXKCAACXKNEEHHEXXEKNXHXNEEBX H H K B KNHXEXXNEKAAHEKAABBXEKCBEAXHKAHXXBEC C C E B KHCBNABXBAEHXHBAHACEXBNBNEXHB B E AC B A A A B KB KXXNKXXNHCXNCBKKAACCKNXHCKBCBNBNXKCN B EB N N XEAKCECANACXHENEBHAXEKXHXBEKB BXXHBA C X H B H H HEK HE B A N K B C A N X E HA B HX B E EX N N E N E A E C E K

Fig. 3 GUI interface of Anfimov table software: The Amfimov table is located in the center of the screen. The character line width is 0.6 mrad according to the test point at the fixed eye position. 
Hou et al.: Display dimming model characterized by three-dimensional ergonomic study

Table 2 Composition of the VF scale.

\begin{tabular}{|c|c|c|c|c|c|}
\hline \multirow{2}{*}{$\frac{\text { Items }}{\text { Blurred vision }}$} & \multicolumn{3}{|c|}{ Not at all- } & \multicolumn{2}{|c|}{ extremely } \\
\hline & 0 & 1 & 2 & 3 & 4 \\
\hline Double vision & 0 & 1 & 2 & 3 & 4 \\
\hline Dry eyes & 0 & 1 & 2 & 3 & 4 \\
\hline Hot or burning eyes & 0 & 1 & 2 & 3 & 4 \\
\hline Not at all irritated eyes & 0 & 1 & 2 & 3 & 4 \\
\hline Sore or aching eyes & 0 & 1 & 2 & 3 & 4 \\
\hline Tired eyes & 0 & 1 & 2 & 3 & 4 \\
\hline
\end{tabular}

Table 3 The components of the VC scale.

\begin{tabular}{|c|c|c|}
\hline Positive & Negative & Citation \\
\hline Cozy & Uncomfortable & $\mathrm{a}, \mathrm{b}, \mathrm{c}$ \\
\hline Harmonious & Incongruity & $d$ \\
\hline Tranquil & Restless & $\mathrm{a}, \mathrm{b}, \mathrm{c}$ \\
\hline Bright & Dim & $\mathrm{a}, \mathrm{b}$ \\
\hline Like & Dislike & $\mathrm{b}$ \\
\hline Natural & Unnatural & $\mathrm{b}$ \\
\hline Lively & Lifeless & $\mathrm{a}, \mathrm{b}$ \\
\hline Clear & Blurred & $\mathrm{a}, \mathrm{c}$ \\
\hline
\end{tabular}

'Vogels' study in 2008.

'Liu's study in 2015.

'Wang's study in 2014.

'Lu's study in 2019.

\subsubsection{Visual fatigue}

The VF scale contains seven sets of descriptions that show significant correlations with VF, which was sensitive in differentiating tracking from reading and monitoring tasks ${ }^{36}$ and identified in a previous study. ${ }^{37}$ A higher score indicates more VF. Table 2 lists the different categories used for assessing fatigue.

\subsubsection{Visual comfort}

The VC scale, shown in Table 3, consists of eight pairs of antonyms chosen from Vogels' questionnaire, and previous studies from Liu et al. and $\mathrm{Lu}$ et al. are referenced for Chinese translations. ${ }^{38-41}$ The eight items inquire on the comfort level, mood, and preferences of the observer in the environment from various angles. Each item is divided into seven levels, and the results are preprocessed by summing the score of each item. A higher score on the VC scale indicates a higher level of comfort.

\section{Results and Statistical Analysis}

Statistical analysis of the raw data is conducted using SPSS Statistics 20 software (IBM). A twoway analysis of variance (ANOVA) is used for the statistical analysis. The following sections 
will elaborate on the experimental results from three aspects: VP, VC, and VF. VP refers to the value of IMC in different conditions; the VC data are collected from the VC scale; the VF data come from the VF scale. Two of the 25 conditions $\left(1 \mathrm{~cd} / \mathrm{m}^{2}\right.$ under 1000 and $\left.2700 \mathrm{~lx}\right)$ are abandoned because the display cannot satisfy the preset parameter value in contrast. Therefore, the data for these two conditions are not included in the analysis process.

\subsection{Visual Performance}

The IMC value is used to represent the VP. The calculation process of the IMC is described in Sec. 2.4.1. Then, an ANOVA was employed to investigate the relationship between VP and lighting conditions. Levene's test reveals the equality of error variances. Table 4 gives the ANOVA results for different dimensions.

Considering the VP as a dependent variable, the ANOVA with the ambient illuminance and display luminance as independent variables show a statistically significant effect of ambient illuminance $(F=2.612, p=0.037)$ and display luminance $(F=51.618, p<0.001)$. In addition, the analysis does not show a significant interactive effect between illuminance and luminance on $\mathrm{VP}(F=0.740, p=0.733)$.

The mean and standard error of the VP under different conditions are shown in Fig. 4. It can be observed that the relationship between ambient illuminance and VP is parabolic under low display luminance conditions. Moreover, the VP exhibits a monotonous increase under high display luminance conditions. As the luminance increases, the growth rate of the VP simultaneously decreases. For different luminance under the same illuminance, the VP exhibits nonlinear monotonic increases in the high luminance area with the appearance of response compression.

Table 4 ANOVA results of VP.

\begin{tabular}{lccccc}
\hline \hline Effect & Type III sum of squares & df & Mean square & $F$ & Sig. \\
\hline$E$ & 0.064 & 4 & 0.016 & 2.612 & 0.037 \\
$L$ & 1.273 & 4 & 0.318 & 51.618 & 0.000 \\
$E \times L$ & 0.064 & 14 & 0.005 & 0.740 & 0.733 \\
\hline \hline
\end{tabular}

Note: $E=$ ambient illuminance; $L=$ display luminance.

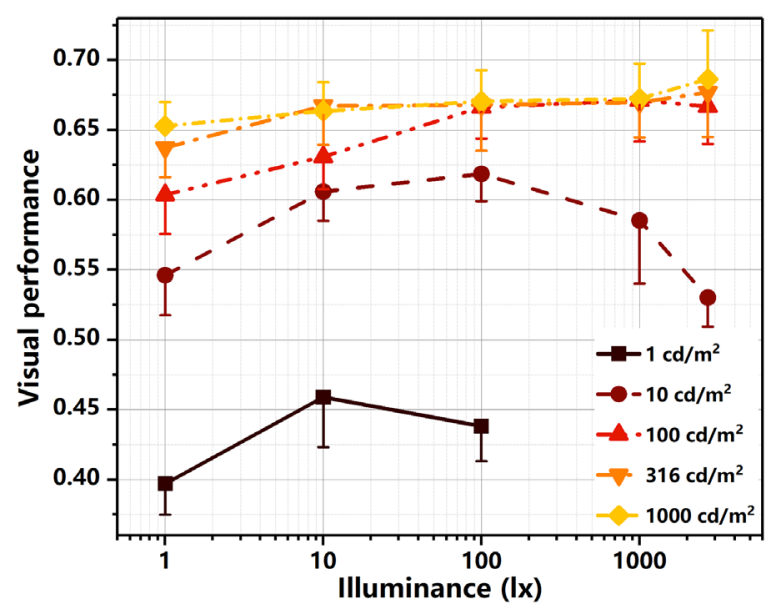

Fig. 4 Mean and standard error of mean (SEM) of the VP with ambient illuminance as the independent variable for five values of display luminance. 
Table 5 ANOVA results for VC.

\begin{tabular}{lccccc}
\hline \hline Effect & Type III sum of squares & df & Mean square & $F$ & Sig. \\
\hline$E$ & 7062.437 & 4 & 1765.609 & 9.827 & 0.000 \\
$L$ & 12220.990 & 4 & 3055.248 & 17.005 & 0.000 \\
$E \times L$ & 8923.150 & 14 & 637.368 & 3.547 & 0.000 \\
\hline \hline
\end{tabular}

Note: $E$ = ambient illuminance; $L=$ display luminance.

\subsection{Visual Comfort}

For the VC, the homogeneity of its variance is tested using Levene's test. The ANOVA, with the ambient illuminance and display luminance as the independent variable and VC as the dependent variable, shows the main effects of ambient illuminance $(F=9.827, p<0.001)$ and display luminance $(F=17.005, p<0.001)$. It also presents the significant interactive effect between illuminance and luminance $(F=3.547, p<0.001)$. It reveals that different combinations of ambient illuminance and display luminance could significantly affect the VC level. Levene's test reveals the equality of error variances. Table 5 lists the ANOVA results for different dimensions.

The mean and standard error of the VC under different conditions are shown in Fig. 5. It can be observed that for the aspects of illuminance and luminance, the comfort level exhibits an upward trend first and then a downward trend; the upward and downward speeds are not consistent. In addition, as the luminance level increases, the peak of the VC curve keeps shifting to the right side of the diagram, which suggests that the optimal illuminance out of $\mathrm{VC}$ outcomes increases.

\subsection{Visual Fatigue}

An ANOVA is conducted on VF for ambient illuminance and display luminance. Prior to this, Levene's test is conducted, and the results reveal the equality of error variances. The ANOVA shows no main effect of the ambient illuminance on VF $(F=0.875, p=0.480)$; however, the display luminance had statistically significant effects on VF $(F=10.547, p<0.001)$. It also shows a significant interactive effect between illuminance and luminance on VF $(F=3.967$, $p<0.001)$. This indicates that different combinations of ambient illuminance and display

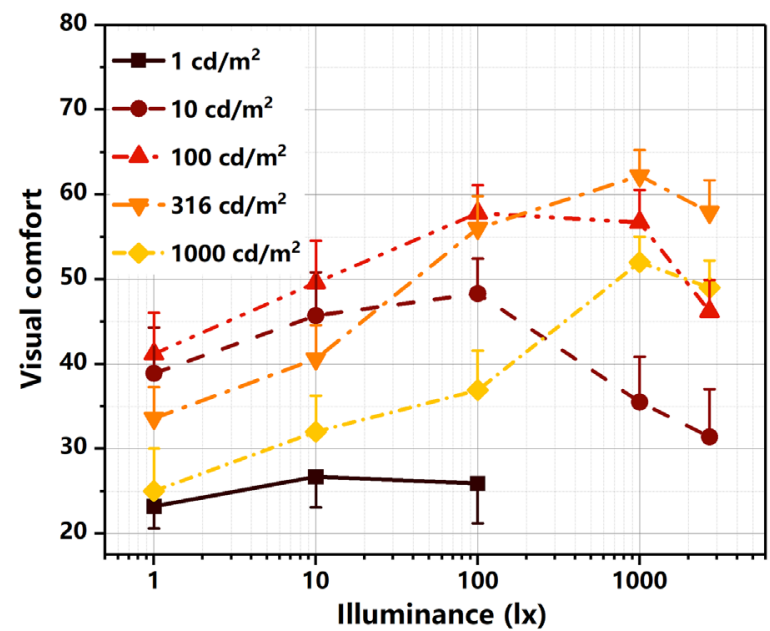

Fig. 5 Mean and standard error (SEM) of the VC with ambient illuminance as the independent variable for five values of display luminance. 
Table 6 ANOVA results of VF.

\begin{tabular}{lccccc}
\hline \hline Effect & Type III sum of squares & df & Mean square & $F$ & Sig. \\
\hline$E$ & 49.587 & 4 & 12.397 & 0.875 & 0.480 \\
$L$ & 597.822 & 4 & 149.455 & 10.547 & 0.000 \\
$E \times L$ & 786.973 & 14 & 56.212 & 3.967 & 0.000 \\
\hline \hline
\end{tabular}

Note: $E=$ ambient illuminance; $L=$ display luminance.

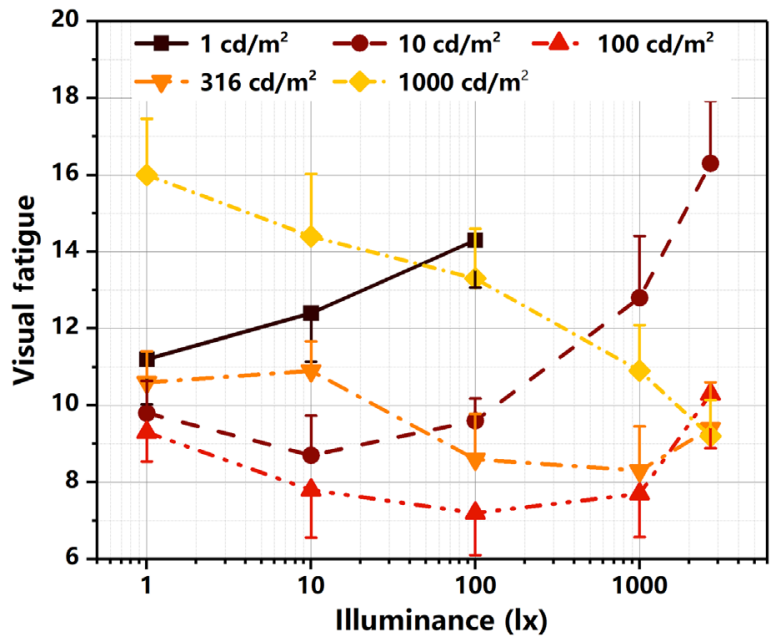

Fig. 6 Mean and standard error (SEM) of the VF with ambient illuminance as the independent variable for five values of display luminance.

luminance may significantly affect VF. Levene's test reveals the equality of error variances. Table 6 lists the ANOVA results for different dimensions.

The mean and standard error of the VF under different conditions are shown in Fig. 6. The three curves of 10,100 , and $316 \mathrm{~cd} / \mathrm{m}^{2}$ show a consistent trend of declining first and then rising. In addition, this trend is asymmetric in the rising and declining sides. It should be noted that the curve of $1 \mathrm{~cd} / \mathrm{m}^{2}$ increases with an increase in illuminance, which indicated that the fatigue increases. Moreover, the curve of $1000 \mathrm{~cd} / \mathrm{m}^{2}$ decreases with an increase in illuminance; i.e., the degree of fatigue decreases.

\section{Establishment of the Display Dimming Model}

In Sec. 3, the data of each condition are analyzed in 3D: VP, VC, and VF. In this section, an integrating dimming curve model based on these dimensions is discussed. The sub-model of each dimension is first fitted based on the data characteristics and theoretical foundation. Subsequently, the analytic hierarchy process (AHP) is employed to construct this 3D evaluation system by calculating the weight of each dimension in the total score (TS); thus, AHP is an effective method to solve the multi-objective comprehensive evaluation. ${ }^{42}$ Finally, the exponential fitting of the TSs is performed to obtain the optimal dimming model in the illuminanceluminance dimension, as shown in Fig. 7.

\subsection{Step1: Establishment of Sub-Model of each Dimension}

According to the Weber-Fechner law, the models are established in the logarithmic system of physical quantities to better match human psychological perception. 


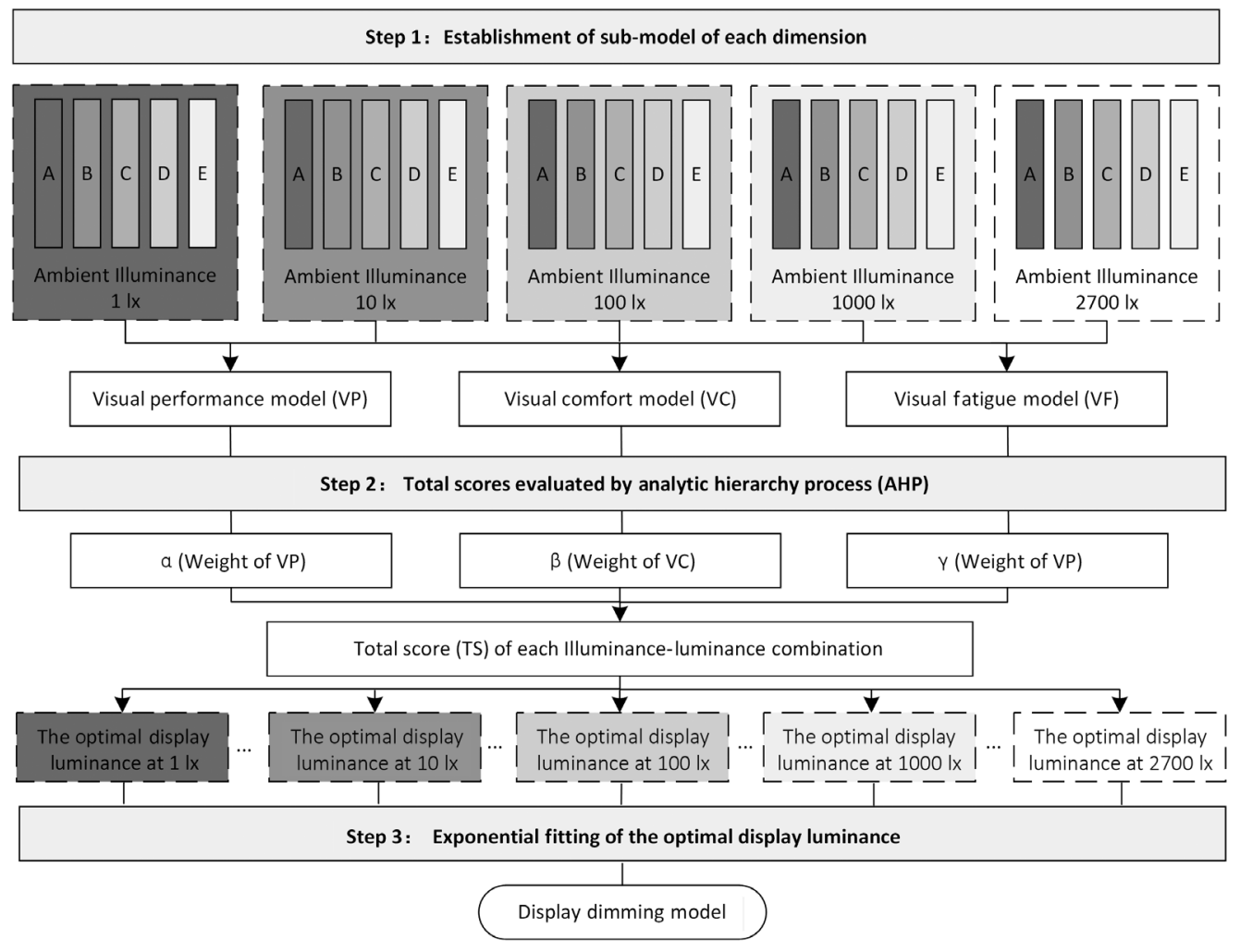

Fig. 7 Model structure and process step. The symbols $A, B, C, D$, and $E$ denote varied luminance level, $A=1 \mathrm{~cd} / \mathrm{m}^{2}, B=10 \mathrm{~cd} / \mathrm{m}^{2}, C=100 \mathrm{~cd} / \mathrm{m}^{2}, D=316 \mathrm{~cd} / \mathrm{m}^{2}, E=1000 \mathrm{~cd} / \mathrm{m}^{2}$, respectively.

\subsubsection{Visual performance model}

Some critical prior knowledge about the VP model was gained in previous studies. Rea and Ouellette $^{43}$ verified a significant linear relationship between VP and the logarithm of retinal illumination. In this study, the relationship between ambient illuminance and VP is parabolic and a monotonous increase under low and high display luminance conditions, respectively. Therefore, it can be assumed that the monotonicity under high luminance is owing to the parameter values being on one side of the parabolic peak. Thus, the relationship between the logarithm of the ambient illuminance and the VP is parabolic. In addition, the response compression of the logarithm of the luminance for VP was confirmed in another study, ${ }^{44}$ which prompted us to conduct the logarithmic transformation based on the logarithm of the luminance. Hence, a twodegree polynomial is used to estimate VP using a least-squares criterion, the illumination value between 1 and $2700 \mathrm{~lx}$, and the luminance value between 1 and $1000 \mathrm{~cd} / \mathrm{m}^{2}$. The polynomial fit is a classical method in psychophysical evaluation with a greater role on issues of design and inference. ${ }^{45}$ The proposed $V P$ model is shown in Eq. (2):

$$
V P=a_{00}+a_{10} E^{\prime}+a_{01} \log _{10} L^{\prime}+a_{20} E^{\prime 2}+a_{11} E^{\prime} \log _{10} L^{\prime},
$$

where $V P$ represents the visual performance, $E^{\prime}$ is the logarithm of the ambient illuminance value, and $L^{\prime}$ is the logarithm of the display luminance value. The coefficient of each term is shown in Table 7. Figure 8 shows a graphical expression of the $V P$ as a function of illuminance and luminance.

The $V P$ model exhibits a good fitting degree with $R^{2}=0.90$, and the Pearson correlation coefficient is 0.87 . The model reveals that the matching degree of display luminance and ambient illuminance impacts VP, even when the contrast is the same. For example, an excessively bright surrounding illuminance is not conducive to VP, even if the readability does not change. 
Hou et al.: Display dimming model characterized by three-dimensional ergonomic study

Table 7 The coefficients of VP, VC, and VF model

\begin{tabular}{lcccccccccc}
\hline \hline Model & $a_{00}$ & $a_{10}$ & $a_{01}$ & $a_{20}$ & $a_{11}$. & $a_{02}$ & $a_{30}$ & $a_{21}$ & $a_{12}$ & $a_{03}$ \\
\hline VP & 0.5854 & 0.04972 & 0.07283 & -0.01249 & 0.008367 & - & - & - & - & - \\
VC & 23.01 & 5.097 & 17.95 & 1.475 & -0.070 & -1.066 & -1.728 & 2.275 & -0.5689 & -1.625 \\
VF & 11.76 & -0.3762 & -2.297 & 0.1498 & 0.1499 & -1.035 & 0.3363 & -0.653 & 0.0664 & 0.7465 \\
\hline \hline
\end{tabular}

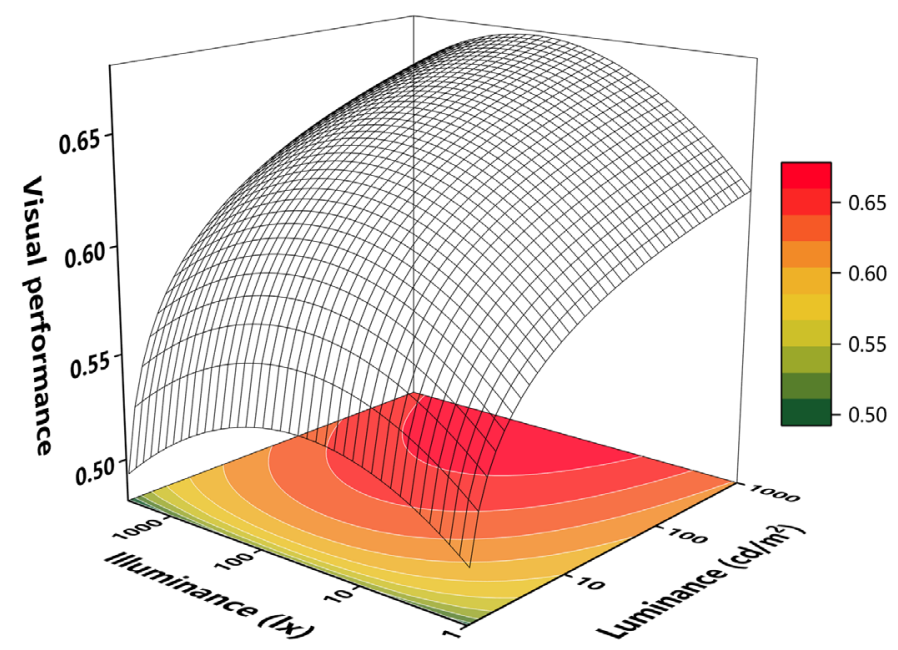

Fig. 8 Curved surface of the VP model. VP is plotted as a function of illuminance and luminance, and both scaled logarithmically.

\subsubsection{Visual comfort model}

From the data trend of rising first and then falling at different speeds, three-degree polynomials are used to estimate VC using a least-squares criterion, an illumination value between 1 and $2700 \mathrm{l} \times$, and a luminance value between 1 and $1000 \mathrm{~cd} / \mathrm{m}^{2}$. The proposed $V P$ model is shown in Eq. (3):

$$
\begin{aligned}
V C= & a_{00}+a_{10} E^{\prime}+a_{01} L^{\prime}+a_{20} E^{\prime 2}+a_{11} E^{\prime} L^{\prime}+a_{02} L^{\prime 2} \\
& +a_{30} E^{\prime 3}+a_{21} E^{\prime 2} L^{\prime}+a_{12} E^{\prime} L^{\prime 2}+a_{03} L^{\prime 3}
\end{aligned}
$$

where $V C$ represents the visual comfort, $E^{\prime}$ is the logarithm of the ambient illuminance value, and $L^{\prime}$ is the logarithm of the display luminance value. The coefficient of each term is shown in Table 7. Figure 9 shows a graphical expression of $V C$ as a function of illuminance and luminance.

The $V C$ model shows an excellent fitting degree with $R^{2}=0.95$, and the Pearson correlation coefficient is 0.92 . The model verifies that the comfortable display luminance increases as the ambient illuminance increases. When there is a mismatch between the ambient illuminance and the display brightness, the user's comfort level will decrease. This mismatch includes highluminance displays under dim ambient illuminance and low-luminance displays under bright ambient illuminance.

\subsubsection{Visual fatigue model}

For VF, the three curves of 10,100 , and $316 \mathrm{~cd} / \mathrm{m}^{2}$ exhibit the three-degree polynomial features with a consistent trend of first decreasing and then rising. Moreover, the curves are asymmetric in the rising and falling sides. However, the 1 and $1000 \mathrm{~cd} / \mathrm{m}^{2}$ curves present a monotonous trend, 


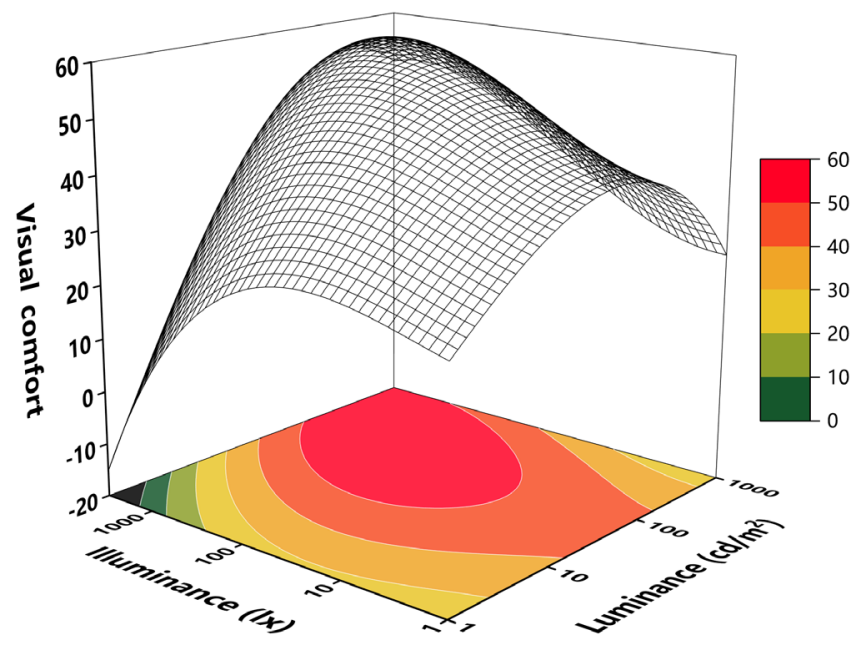

Fig. 9 Curved surface of the VC model. VC is plotted as a function of illuminance and luminance, and both scaled logarithmically.

suggesting that the valleys are not in the parameter range. Hence, three-degree polynomials are also used to estimate VF using a least-squares criterion, the illumination value between 1 and $2700 \mathrm{~lx}$, and the luminance value between 1 and $1000 \mathrm{~cd} / \mathrm{m}^{2}$. The proposed $V F$ model is shown in Eq. (4):

$$
\begin{aligned}
V F= & a_{00}+a_{10} E^{\prime}+a_{01} L^{\prime}+a_{20} E^{\prime 2}+a_{11} E^{\prime} L^{\prime}+a_{02} L^{\prime 2} \\
& +a_{30} E^{\prime 3}+a_{21} E^{\prime 2} L^{\prime}+a_{12} E^{\prime} L^{\prime 2}+a_{03} L^{\prime 3},
\end{aligned}
$$

where $V F$ denotes the visual fatigue, $E^{\prime}$ is the logarithm of the ambient illuminance value, and $L^{\prime}$ is the logarithm of the display luminance value. The coefficient of each term is shown in Table 7. Figure 10 shows a graphical expression of $V F$ as a function of illuminance and luminance.

The $V F$ model shows an excellent fitting degree with $R^{2}=0.96$, and the Pearson correlation coefficient is 0.94 . The model indicates that an appropriate matching relationship between ambient illuminance and the display luminance led to a low degree of VF, as shown in the green area in Fig. 10. However, when they did not match, severe VF was caused.

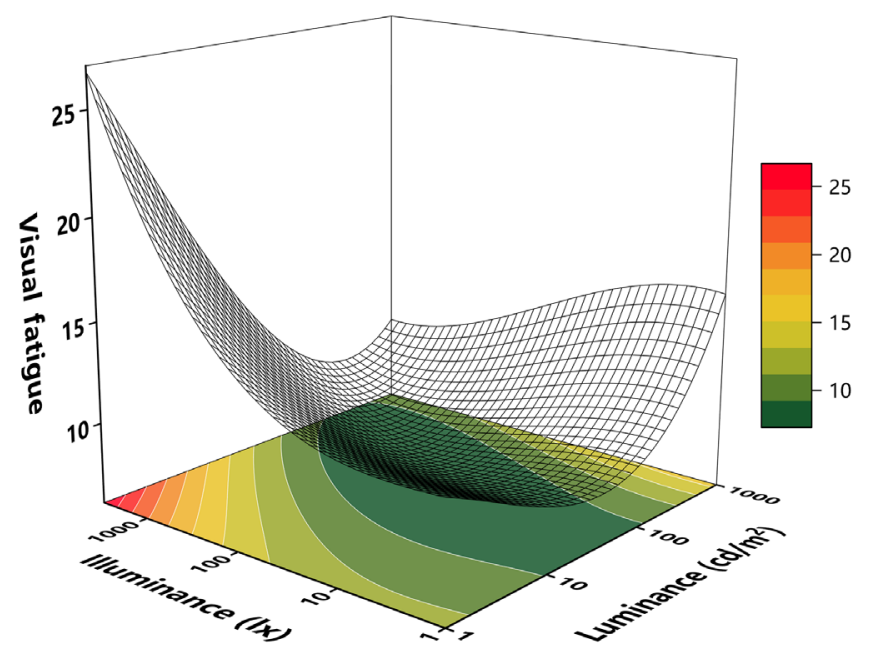

Fig. 10 Curved surface of the VF model. VF is plotted as a function of illuminance and luminance, and both scaled logarithmically. 


\subsection{Step2: Total Scores Evaluated by Analytic Hierarchy Process}

AHP is employed to construct an evaluation system by calculating the weight of each dimension in the TS. First, a hierarchical model is built, as shown in Fig. 7. Optimal display luminance is the target layer of the model, and the indicator layer consists of 3D: VP, VC, and VF. The casing layer consists of conditions with different illuminance-luminance combinations.

The data must be preprocessed to be dimensionless and formed the relative response values ${ }^{44}$ by dividing the absolute response value by the maximum response value (within the parameters of this experiment), before entering the model. The preprocessing functions are shown in Eqs. (5)-(7):

$$
\begin{gathered}
V P^{R}=V P / V P_{\max }, \\
V C^{R}=V C / V C_{\max }, \\
V F^{R}=1-V F / V F_{\max },
\end{gathered}
$$

where $V P^{R}$ represents the relative $\mathrm{VP}, V P_{\max }=0.7 ; V C^{R}$ represents the relative $\mathrm{VC}$, $V C_{\max }=0.760 ; V F^{R}$ represents the relative $V F, V F_{\max }=27$. It should be noted that a higher $\mathrm{VF}(V F)$ value in Eq. (4) generates a more negative fatigue. Therefore, the relative VF is defined by Eq. (7), where a value of $V F^{R}$ closer to 1 indicates less fatigue with a positive effect.

According to this framework, $\mathbf{A}$ is defined as the index matrix, which consists of the relative VP score $V P^{R}$, relative VC score $V C^{R}$, and relative VF score $V F^{R}$. In addition, the weights corresponding to the three indicators are $\alpha, \beta$, and $\gamma$, which is defined as the weight matrix W. Finally, the product of $A$ and $w$ is the $T S$. Thus, the $T S$ of each condition can be expressed as

$$
\mathbf{A}=\left[V P^{R}, V C^{R}, V F^{R}\right]^{\mathrm{T}} \quad \mathbf{W}=[\alpha, \beta, \gamma] \quad T S=\mathbf{W A} .
$$

To obtain the weight matrix $\mathbf{W}$, a paired comparison matrix $\mathbf{X}$ is constructed; the element $x_{i j}$ in the matrix represents the ratio of the degree of influence of the $i$ 'th element of $\mathbf{A}$ to the target layer relative to the $j$ 'th element: ${ }^{46}$

$$
\mathbf{X}=\left(x_{i j}\right)_{3 \times 3} .
$$

The pairwise comparison matrix data are obtained through expert investigation weight method $^{37}$ by employing 33 pilots from Airlines Company. All the pilots have more than $100 \mathrm{~h}$ of flight experience. The expert investigation questionnaire consisted of six items. A rating of

\begin{tabular}{|c|c|c|c|c|c|c|c|c|c|c|c|c|c|c|c|c|c|c|}
\hline Element $i$ & & & & & & & & & & & & & & & & & & Element $j$ \\
\hline VP & 9 & 8 & 7 & 6 & 5 & 4 & 3 & 2 & 1 & $1 / 2$ & $1 / 3$ & $1 / 4$ & $1 / 5$ & $1 / 6$ & $1 / 7$ & $1 / 8$ & $1 / 9$ & VF \\
\hline VP & 9 & 8 & 7 & 6 & 5 & 4 & 3 & 2 & 1 & $1 / 2$ & $1 / 3$ & $1 / 4$ & $1 / 5$ & $1 / 6$ & $1 / 7$ & $1 / 8$ & $1 / 9$ & VC \\
\hline VF & 9 & 8 & 7 & 6 & 5 & 4 & 3 & 2 & 1 & $1 / 2$ & $1 / 3$ & $1 / 4$ & $1 / 5$ & $1 / 6$ & $1 / 7$ & $1 / 8$ & $1 / 9$ & VC \\
\hline VF & 9 & 8 & 7 & 6 & 5 & 4 & 3 & 2 & 1 & $1 / 2$ & $1 / 3$ & $1 / 4$ & $1 / 5$ & $1 / 6$ & $1 / 7$ & $1 / 8$ & $1 / 9$ & VP \\
\hline VC & 9 & 8 & 7 & 6 & 5 & 4 & 3 & 2 & 1 & $1 / 2$ & $1 / 3$ & $1 / 4$ & $1 / 5$ & $1 / 6$ & $1 / 7$ & $1 / 8$ & $1 / 9$ & VP \\
\hline VC & 9 & 8 & 7 & 6 & 5 & 4 & 3 & 2 & 1 & $1 / 2$ & $1 / 3$ & $1 / 4$ & $1 / 5$ & $1 / 6$ & $1 / 7$ & $1 / 8$ & $1 / 9$ & VF \\
\hline
\end{tabular}
$1 / 9-1-9$ is used for each item, ${ }^{42,46}$ where " 1 " means the $i$ ' th element has the same influence as the $j$ 'th element, " $1 / 9$ " means that the $j$ 'th element has a stronger influence than the $i$ 'th element, and "9" means that the $i$ 'th element has a stronger influence than the $j$ 'th element. The pilots were

Table 8 Composition of the expert investigation questionnaire. 
Table 9 Weights of each dimension by expert investigation weight method.

\begin{tabular}{lccc}
\hline \hline & VP $(\alpha)$ & VC $(\beta)$ & VF $(\gamma)$ \\
\hline Weight & 0.33 & 0.40 & 0.27 \\
\hline \hline
\end{tabular}

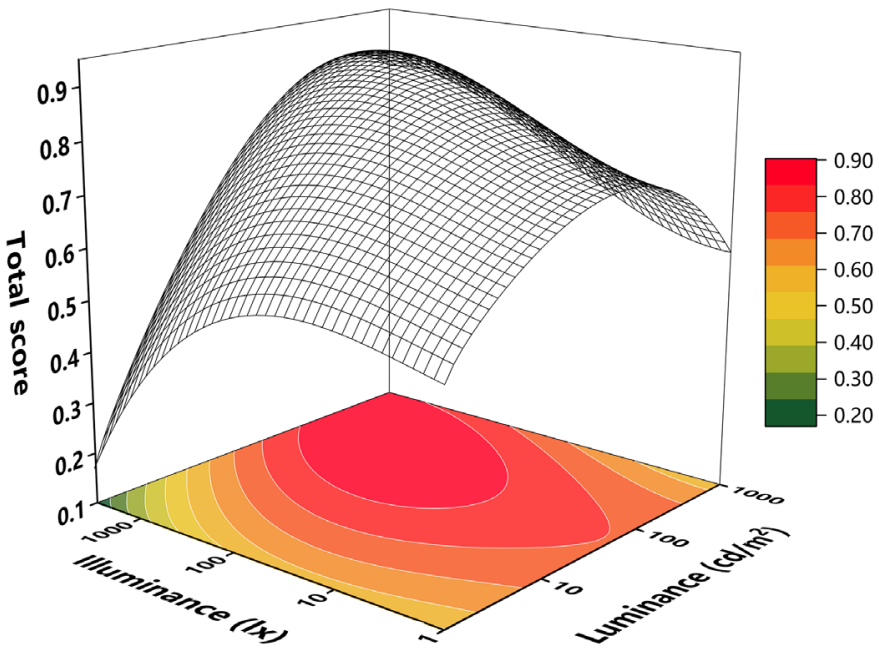

Fig. 11 Curved surface of the TS model. The TS is plotted as a function of illuminance and luminance, and both scaled logarithmically.

asked to answer the questionnaire according to their feelings during flight. The composition of the expert investigation questionnaire is shown in Table 8.

The consistency of the questionnaires was tested by the consistency ratio. ${ }^{42,46}$ For those 18 questionnaires, whose CR were smaller than 0.1 , were adopted for further determining the weight vector matrix by the characteristic root method. ${ }^{47}$ The mean results of the weights of each dimension are shown in Table 9.

The TS is calculated using Eq. (8). It can be observed from Fig. 11 that the areas with higher TSs are approximately distributed on the diagonal of the logarithmic coordinate system. This implies that with an increase in ambient illuminance, the display luminance should be correspondingly increased to fulfill the comprehensive needs of the observer in $3 \mathrm{D}$.

\subsection{Step3: Exponential Fitting of the Illuminance-Luminance Dimming Model}

The TS [based on Eq. (8)] obtained in the second step is the basis for identifying the optimal combination of illuminance and luminance. The 0-1 standardization of TS different luminance under each illuminance is processed to find the relatively optimal luminance for each illuminance. The standardized results are shown in Fig. 12, and an area with a relative TS (RTS) > 0.9 is defined as the optimal area. Moreover, an exponential fitting is performed for the highest RTS (RTS $=1$ ) in illuminance-luminance dimensions, and the dimming model is built as follows:

$$
L=5.464 E^{0.4922}+41
$$

where $E$ is the ambient illuminance value, and $L$ is the display luminance value. The dimming model provides a foundation for adaptive dimming curves for cockpit displays and monitors at work and in daily life. 


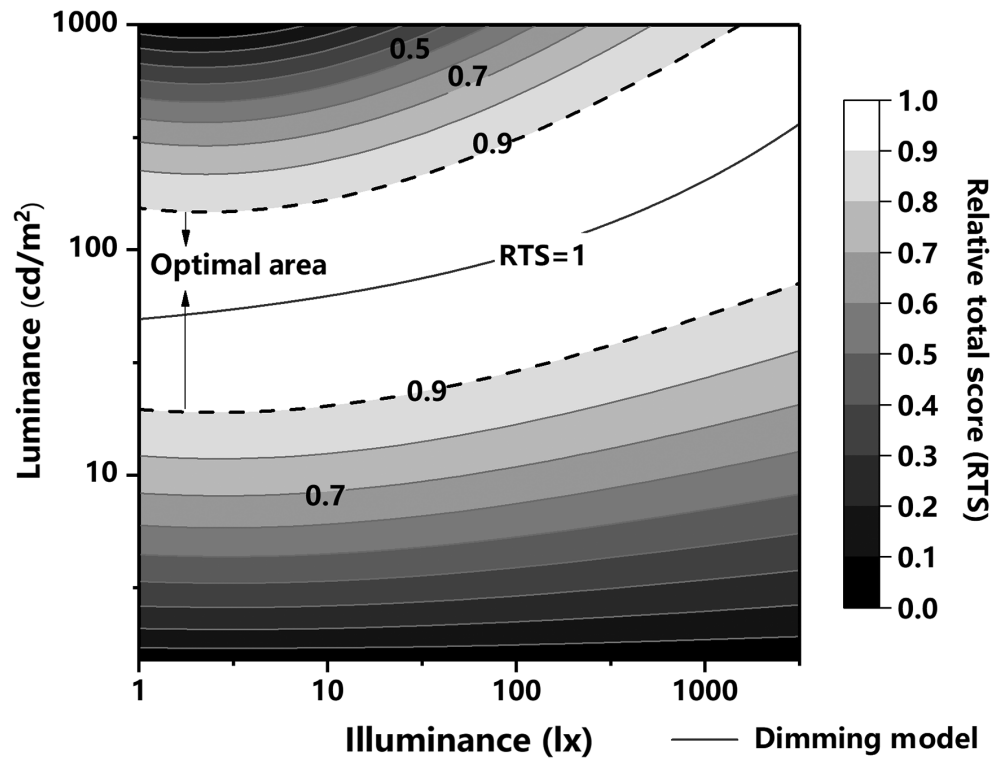

Fig. 12 Recommended display diming model in this experiment. The white area in the middle of the graph indicates that the RTS is $>0.9$; thus, it is defined as the optimal area. The black curve in the optimal area is the dimming model [based on Eqs. (4)-(9), RTS =1]. The gradient area on both sides is divided according to the contour line of the RTS. A darker color indicates a lower RTS.

\section{Validation of the Display Dimming Model}

The established model in Sec. 4 is validated with the earlier relative studies, by $\mathrm{Xu}$ and $\mathrm{Zhu}{ }^{2}$ Lin and Huang, ${ }^{14}$ Buchner et al., ${ }^{21}$ etc. ${ }^{1,16,48}$ The recommended values or models of display luminance under different ambient illumination through published ergonomic studies are included in the verification dataset for this model. The details of the model verification dataset are shown in Table 10 and Fig. 13. The results showed good validation to the degree that most of the combinations of ambient illuminance and display luminance in validation dataset are covered in the optimal area by our proposed model.

Table 10 The details of the model verification dataset.

\begin{tabular}{|c|c|c|c|c|c|c|c|}
\hline Profiles & Subjects & Age & $\begin{array}{l}\text { Display } \\
\text { polarity }\end{array}$ & $\begin{array}{l}\text { Optimization } \\
\text { goal }\end{array}$ & $\begin{array}{l}\text { Lighting } \\
\text { conditions }\end{array}$ & Recommendations & $\begin{array}{l}\text { Within the } \\
\text { optimal } \\
\text { area of the } \\
\text { proposed } \\
\text { model }\end{array}$ \\
\hline $\begin{array}{l}\text { (Xu and } \\
\text { Zhu, 1990) }\end{array}$ & $n=9$ & 17 to 34 & Negative & VP & $800 \mathrm{~lx}$ & $90 \mathrm{~cd} / \mathrm{m}^{2}$ & Yes \\
\hline $\begin{array}{l}\text { (Lin and } \\
\text { Huang, 2006) }\end{array}$ & $n=28$ & $\begin{array}{l}\text { College } \\
\text { students }\end{array}$ & Positive & VP & $\begin{array}{l}200 \text { to } \\
800 \mathrm{~lx}\end{array}$ & $100-200 \mathrm{~cd} / \mathrm{m}^{2}$ & Yes \\
\hline $\begin{array}{l}\text { (Buchner } \\
\text { et al. 2009) }\end{array}$ & $n=124$ & 18 to 55 & $\begin{array}{l}\text { Positive and } \\
\text { Negative }\end{array}$ & VP & $550 \mathrm{~lx}$ & $77 \mathrm{~cd} / \mathrm{m}^{2}$ & Yes \\
\hline $\begin{array}{l}\text { (Na and } \\
\text { Suk, 2015) }\end{array}$ & $n=50$ & $\begin{array}{c}\text { MSD }= \\
21.76 \pm 3.28\end{array}$ & Positive & $\begin{array}{c}\text { Visual } \\
\text { Preference }\end{array}$ & $<1 \mathrm{Ix}$ & $40 \mathrm{~cd} / \mathrm{m}^{2}$ & Yes \\
\hline $\begin{array}{l}\text { (Kim et al. } \\
2018)^{1}\end{array}$ & $n=6$ & $M=25.2$ & Positive & VP & $\begin{array}{l}50 \text { to } \\
500 \mathrm{~lx}\end{array}$ & $\begin{array}{c}200 \text { to } \\
500 \mathrm{~cd} / \mathrm{m}^{2}\end{array}$ & Mostly \\
\hline
\end{tabular}




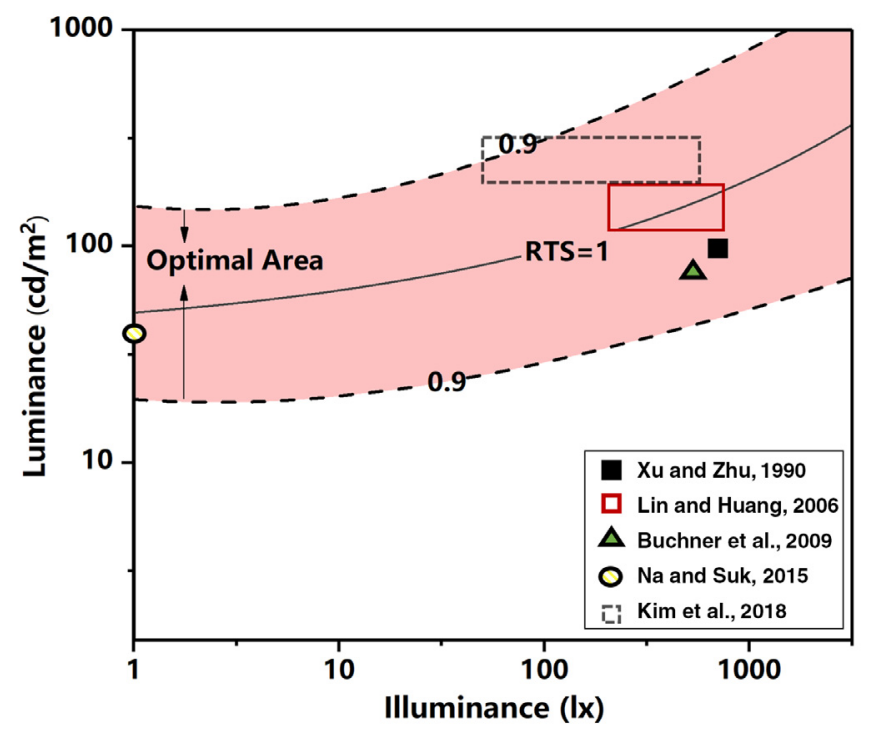

Fig. 13 Validation of the proposed model by earlier studies. ${ }^{1,2,14,16,21}$

\section{Discussion}

This study is based on a three-dimensional ergonomics study, which investigates the effects of different display luminance under different ambient illuminance on the user's comprehensive outcomes. The research results are divided into two parts. The first part is a separate analysis of the effects of the 3D. This leads us to recognize the degree and significance of the impact of the illuminance and luminance on a specific index. The second part is the integration of the 3D. On this basis, the TS model is constructed for each ambient illuminance, and a wide-range dimming model is then obtained. The discussion of these two parts is presented separately in the following subsections.

\subsection{Effects of Ambient Illuminance and Display Luminance on each Dimension}

For the VP part, the results of this study show that both the display luminance and ambient illuminance have significant impacts on VP, even when the contrast is the same. The relationship between the logarithm of the ambient illuminance and the VP is parabolic. This phenomenon may be owing to the change in the contrast threshold of the human eye with ambient lighting intensity. This implies that although the contrast of the display remains constant, the VP declines when the ambient illuminance increases and the threshold increment of the contrast decreases because the contrast threshold also increases. For a different luminance under the same illuminance, the VP exhibits an increase with an increase in luminance. Additionally, it reveals the response compression in the high-illuminance area. In previous studies, only the ambient illumination or display luminance was used to find the optimal solution under particular conditions. ${ }^{4,5}$ For example, Shen et al. ${ }^{4}$ showed that search speed on an electronic paper display increased with an increase in illumination from $300 \mathrm{~lx}$ (45.6 s), $700 \mathrm{~lx}$ (44.18 s) to $1500 \mathrm{~lx}$ (43.24 s). Lin ${ }^{5}$ showed that illumination intensity significantly affects character identification performance, which was better at $500 \mathrm{~lx}$ and $800 \mathrm{~lx}$ than $200 \mathrm{~lx}$. Lin and Huang ${ }^{26}$ study proved that the normal white ambient lighting condition with $500 \mathrm{~lx}$ was the optimal condition accompanied by a display with a primary background. However, these optimal results were obtained under specific illuminance or luminance conditions. In a real application environment, the environmental illuminance is often changed within a range; thus, these results cannot fully support the dimming of the display. Lin and Huang ${ }^{14}$ study in 2006 attempted to solve this problem. The variable was set as the combination of screen luminance and ambient illumination, display 
luminance ranged from 3.1 to $200.0 \mathrm{~cd} / \mathrm{m}^{2}$, and the ambient illumination from 200 to $800 \mathrm{~lx}$. The study indicated that character identification under relatively high ambient illumination is more affected by the background luminance of the screen than by the contrast ratio or contrast sensitivity. In contrast, the parameter range of the present study is broader (the display luminance and ambient illumination range from 1 to $1000 \mathrm{~cd} / \mathrm{m}^{2}$ and 1 to $2700 \mathrm{~lx}$, respectively), and the parameters are set equidistantly in logarithmic coordinates according to the human body's perception characteristics of physical quantities. Our study shows the consistent conclusion that the readability of negative displays is affected by the display luminance when the contrast remains constant and obtains optimal display luminance, which can induce the best VP under different ambient illumination.

For the VC part, the present study identifies that the ambient illuminance and display luminance reveal interaction effects on VC. Wang et al. ${ }^{25}$ collected the subjective comfort evaluation of subjects observing three-dimensional pictures. They indicated that the "highest luminance" of $47.3 \mathrm{~cd} / \mathrm{m}^{2}$ performed better than the low luminance $\left(0.38 \mathrm{~cd} / \mathrm{m}^{2}\right)$ for ambient illuminance values of both $55 \mathrm{~lx}$ and $300 \mathrm{~lx}$. Based on the luminance level in their study, they inferred that a brighter display luminance creates a more comfortable feeling for viewers. However, this conclusion is one-sided because they did not set a high range for the display luminance; thus, the negative effect of excessively bright luminance was not identified. Huang and Menozzi ${ }^{49}$ showed that discomfort glare impairs peripheral VP in the attending stimulus in a virtual reality environment. When the display luminance is too high, it becomes a glare light source, which affects the comfort of the visual environment. The discomfort caused by the low luminance of the display is primarily owing to the anxiety over not being able to find a target quickly. ${ }^{50} \mathrm{Na}$ and Suk ${ }^{16}$ showed that people preferred a smartphone screen at $40 \mathrm{~cd} / \mathrm{m}^{2}$ with an ambient illuminance below 1 lx. This is consistent with the optimal area in Fig. 11 . However, $55 \mathrm{~cd} / \mathrm{m}^{2}$ is the optimal value when compared with the recommended dimming curve in this study, and the difference may be caused by the experimental design and data analysis. They did not set an intermediate value between 40 and $70 \mathrm{~cd} / \mathrm{m}^{2}$, and concluded by directly using the relative optimal luminance among these values. In addition, Lin et al. ${ }^{51}$ investigated the impact of overall display luminance on VC in long-distance $(5 \mathrm{~m})$ observation. The results showed that the relationship between the comfort rating and luminance is parabolic. This is very similar to the relationship identified in this study. The difference is that this study employs a third-degree polynomial to fit the luminance and $\mathrm{VC}$ because the comfort rating declines faster when the luminance is higher than the optimal value. This suggests that an excessively bright display can cause the glare phenomenon to affect VC. In general, this study explores the optimal combination of display luminance and ambient illuminance on a larger scale, and forms a quantitative $\mathrm{VC}$ model on this basis.

For the VF part, this experiment indicates that the display luminance primarily affects VF. Lee and Whang ${ }^{6}$ showed that VF increased slowly with an increase in the display luminance, particularly when the display luminance is low. However, at a high luminance level, for which the luminance of still images was higher than $400 \mathrm{~cd} / \mathrm{m}^{2}$, the viewer felt intense VF. However, Chang et al. ${ }^{52}$ indicated that the display medium and ambient illuminance had no significant effects on participants' subjective VF. These differences may have occurred because the displays employed in the experiments were all electronic paper displays, which are not self-luminous devices.

\subsection{Wide-Range Dimming Model based on Three Dimensions}

According to the results of each dimension, it can be observed that the optimal conditions for the 3D are varied. For example, high display luminance can contribute to VP; however, it simultaneously causes severe VF and low VC. This regular can be explained by the Pearson correlation analysis results of the data in this study, which shows that a positive correlation between VP and VC with $r=0.351(p<0.01)$, a negative correlation between VP and VF with $r=-0.188$ $(p<0.01)$, and a negative correlation between VC and VF with $r=-0.551 \quad(p<0.01)$. Although the results show significant meaning $(p<0.01)$, it is not a high correlation degree; that means exploring the optimal combination is a complex optimization problem and all the $3 \mathrm{D}$, including VP, comfort levels, and VF, should be considered for the dimming model. 
In this study, sub-models of each dimension are constructed to clarify their characteristics. Moreover, a comprehensive optimal display dimming model is built in the illuminanceluminance dimension by calculating the weight of each dimension in the TS. This study provides a basis for solving the problem of complex light environment matching.

However, this study is based on the negative polarity display, and the applicability of the positive polarity display needs to be further investigated. The parameter range can also be expanded in future studies. In addition, the participants in this study are in a small age range from 22 to 26 . Due to the ocular structural changes by age, ${ }^{53}$ a larger age range inclusion criterion should be considered in future studies.

\section{Conclusions}

This study proposes a dimming model integrating VP, VC, and VF to set proper screen luminance and ambient illumination. The effects of different combinations of ambient illumination and display luminance on human performance, comfort, and VF were investigated. Moreover, a sub-model of each dimension is constructed to clarify their features. Based on the data analysis, the following conclusions can be drawn:

- Both the ambient illuminance and the display luminance can significantly impact VP, even when the contrast remains constant.

- The ambient illuminance and display luminance exhibit interaction effects on VC and VF. Thus, the display luminance should vary with the ambient illumination.

- A wide range (1 to $2700 \mathrm{~lx}$ ) dimming model based on 3D is built, which provides a theoretical foundation to set the dimming curve of the display devices.

\section{Acknowledgments}

This research is supported by the National Key R\&D Program of China (Project No. 2017YFB0403703). The authors declare no conflicts of interest.

\section{References}

1. M. Kim et al., "Optimum display luminance depends on white luminance under various ambient illuminance conditions," Opt. Eng. 57, 024106 (2018).

2. W. Xu and Z. X. Zhu, "The effects of ambient illumination and target luminance on color coding in a CRT display," Ergonomics 33, 933-944 (1990).

3. C. C. Lin, "Effects of screen luminance combination and text color on visual performance with TFT-LCD," Int. J. Ind. Ergon. 35, 229-235 (2005).

4. I. H. Shen et al., "Lighting, font style, and polarity on visual performance and visual fatigue with electronic paper displays," Displays 30, 53-58 (2009).

5. C.-C. Lin, "Effect of noise intensity and illumination intensity on visual performance," Percept. Mot. Skills 119, 441-454 (2014).

6. H. Lee and K. W. Whang, "A quantitative measurement of LCD and PDP TVs for human visual preference and fatigue," Displays 33, 1-6 (2012).

7. P.-C. Wang et al., "Luminance effects influencing perception of 3-D TV imagery," J. Soc. Inf. Disp. 20, 197-207 (2012).

8. H. L. Yi et al., "Luminance determination of CPA and PBAs in aircraft cockpits based on ergonomic research," Appl. Mech. Mater. 602-605, 791-794 (2014).

9. B. A. Yang, Y. D. Lin, and Y. J. Sun, "Transient effects of harsh luminous conditions on the visual performance of aviators in a civil aircraft cockpit," Appl. Ergon. 44, 185-191 (2013).

10. R. G. Rodriguez and A. Pattini, "Effects of a large area glare source in cognitive efficiency and effectiveness in visual display terminal work," Leukos 8, 283-299 (2012).

11. T. A. Ranney, L. A. Simmons, and A. J. Masalonis, "Prolonged exposure to glare and driving time: effects on performance in a driving simulator," Accid. Anal. Prev. 31, 601 (1999). 
12. W. K. E. Osterhaus and I. L. Bailey, "Large area glare sources and their effect on visual discomfort and visual performance at computer workstations," Ind. Appl. Soc. Meeting.

13. J. J. Gallimore and J. M. Stouffer, "Research and development toward automatic luminance control of electronic displays," Int. J. Aviat. Psychol. 11, 149-168 (2001).

14. C. C. Lin and K. C. Huang, "Effects of ambient illumination and screen luminance combination on character identification performance of desktop TFT-LCD monitors," Int. J. Ind. Ergon. 36, 211-218 (2006).

15. F. Leccese et al., "Lighting assessment of ergonomic workstation for radio diagnostic reporting," Int. J. Ind. Ergon. 57, 42-54 (2017).

16. N. Na and H. J. Suk, "Adaptive display luminance for viewing smartphones under low illuminance," Opt. Express 23, 16912-16920 (2015).

17. K. De Moor et al., "Influence of high ambient illuminance and display luminance on readability and subjective preference," Proc. SPIE 9394, 93941E (2015).

18. C. Piepenbrock, S. Mayr, and A. Buchner, "Smaller pupil size and better proofreading performance with positive than with negative polarity displays," Ergonomics 57, 1670-1677 (2014).

19. D. Bauer and C. R. Cavonius, "Improving the legibility of visual display units through contrast reversal," in Ergonomic Aspects of Visual Display Terminals, E. Grandjean and E. Vigliani, Eds., pp. 137-142, Taylor and Francis, London (1980).

20. S. Berman et al., "Luminance-controlled pupil size affects word-reading accuracy," J. Illum. Eng. Soc. 25, 51-59 (1996).

21. A. Buchner, S. Mayr, and M. Brandt, "The advantage of positive text-background polarity is due to high display luminance," Ergonomics 52, 882-886 (2009).

22. A. Creed, I. Dennis, and S. Newstead, "Effects of display format on proofreading witH VDUS," Behav. Inf. Technol. 7, 467-478 (1988).

23. I. Moreno, "Illumination uniformity assessment based on human vision," Opt. Lett. 35, 4030-4032 (2010).

24. S. Sekine, "Spectral distributions of clear sky light and their chromaticities," Journal of Light and Visual Environment 15, 23-32 (1991).

25. P. C. Wang et al., "The effect of stereoscopic display luminance and ambient illuminance on physiological measurement and image quality," Proc. SPIE 8043, 80430D (2011).

26. C.-C. Lin and K.-C. Huang, "Effects of ambient illumination conditions and background color on visual performance with TFT-LCD screens," Displays 34, 276-282 (2013).

27. B. H. Crawford, "Visual adaptation in relation to brief conditioning stimuli," Proc. R. Soc. B Biol. Sci. 134, 283-302 (1947).

28. L. Poot, H. P. Snippe, and J. H. V. Hateren, "Dynamics of adaptation at high luminances: adaptation is faster after luminance decrements than after luminance increments," $\mathrm{J}$. Opt. Soc. Am. A 14, 2499-2508 (1997).

29. F. M. Thoss et al., "Subjective and electroretinographic dynamics of light adaptation in the human visual system," Vision 2, 10 (2018).

30. CIE, ILV: International Lighting Vocabulary, CIE, Vienna (2011).

31. X. Zhang, Child and Adolescent Hygiene, Science Press, Beijing (2009).

32. Y. H. Yan et al., "Productivity and physiological response of students subjected to fluorescent lamps with different colour temperatures and luminance level," J. Civil Archit. Environ. Eng. 32(4), 85-89 (2010).

33. M. Khoroshukha et al., "Influence of blood types serologic markers on development of concentration function of young 13-16 year old athletes," J. Phys. Educ. Sport 18, 1890-1895 (2018).

34. X. Zhao et al., "The effect of stroboscopic effect on human health indicators," Light. Res. Technol. 52(3), 389-406 (2020).

35. M. Huang et al., "Influence of the parameters of light source on observers' visual performance," Appl. Mech. Mater. 731, 124-128 (2015).

36. C.-F. Chi and F.-T. Lin, "A comparison of seven visual fatigue assessment techniques in three data-acquisition VDT tasks," Hum. Factors 40, 577-590 (1998).

37. Y. Wang et al., "A model for evaluating visual fatigue under LED light sources based on long-term visual display terminal work," Light. Res. Technol. 50, 729-738 (2018). 
38. H. H. Wang et al., "A study of atmosphere perception of dynamic coloured light," Light. Res. Technol. 46, 661-675 (2014).

39. I. Vogels, "Atmosphere metrics," in Probing Experience: From Assessment of User Emotions and Behaviour to Development of Products, J. H. D. M. Westerink et al., Eds., pp.25-41, Springer, Dordrecht, Netherlands (2008).

40. X. Y. Liu, M. R. Luo, and H. Li, "A study of atmosphere perceptions in a living room," Light. Res. Technol. 47, 581-594 (2015).

41. Y. Lu et al., "Impacts of LED dynamic white lighting on atmosphere perception," Light. Res. Technol. 51, 1143-1158 (2019).

42. T. L. Saaty, "Decision making with the analytic hierarchy process," Int. J. Serv. Sci. 1, 83 (2008).

43. M. S. Rea and M. J. Ouellette, "Visual performance using reaction times," Light. Res. Technol. 20, 139-153 (1988).

44. M. S. Rea and M. J. Ouellette, "Relative visual performance: a basis for application," Light. Res. Technol. 23, 135-144 (1991).

45. K. Smith, "On the standard deviations of adjusted and interpolated values of an observed polynomial function and its constants and the guidance they give towards a proper choice of the distribution of observations," Biometrika 12, 1-85 (1918).

46. T. L. Saaty, "Axiomatic foundation of the analytic hierarchy process," Manage. Sci. 32, 841-855 (1986).

47. S. Ren, "Assessment on logistics warehouse fire risk based on analytic hierarchy process," Procedia Eng. 45, 59-63 (2012).

48. K. Seung-Ryeol et al., "Optimum display luminance dependence on ambient illuminance," Opt. Eng. 56, 017110 (2017).

49. Y. Y. Huang and M. Menozzi, "Effects of discomfort glare on performance in attending peripheral visual information in displays," Displays 35, 240-246 (2014).

50. Y. D. Lin et al., "Predicting the effects of discomfort glare on visual performance of pilots during nighttime flying," Proc. Int. Conf. Mechatron., Control and Electron. Eng., Advances in Intelligent Systems Research, L. Yaofei, C. Guiran, and L. Zhen, Eds., Vol. 113, pp. 490-494 (2014).

51. M. W. Lin et al., "Flicker-glare and visual-comfort assessments of light emitting diode billboards II: color display," Appl. Opt. 54, 4089 (2015).

52. P. C. Chang, K. K. Shieh, and S. Y. Chou, "Reading performance and visual fatigue when using electronic paper displays in long-duration reading tasks under various lighting conditions," Displays 34, 208-214 (2013).

53. H.-P. Huang, L.-C. Ou, and Y. Yuan, "Effects of age and ambient illuminance on visual comfort for reading on a mobile device," Color Res. Appl. 42, 352-361 (2017).

Dandan Hou received her BE degree at the College of Automation, Harbin Engineering University, in 2016. She is currently pursuing a $\mathrm{PhD}$ at the Department of Light Sources and Illuminating Engineering, Fudan University, Shanghai, China. Her research interests include human factors in lighting, color vision, and human centric lighting. She has been awarded many funding projects, including the Special Project for China Commercial Aircraft and National Key RandD Program of China.

Wei Xu received her BS and MS degrees at Zhejiang University in 1997 and 2000, respectively, and a $\mathrm{PhD}$ from Fudan University, Shanghai, China, in 2011. She is now an associate research fellow of the Department of Light Sources and Illuminating Engineering, Fudan University. Her research interests include visual and non-visual effects of lighting, lighting optical system analysis, and photoirradiation measurement.

Shuo Jing received her BS degree at the School of Mechanical Engineering, Shanghai Institute of Technology, Shanghai, China, in 2014. From 2015 to 2017, she was a structural engineer of lamp in PN.STONE. From 2017 to 2020, she was research assistant in the Department of Light Sources and Illuminating Engineering, Fudan University. Her research interest includes simulation and optimization for lighting systems. 
Yandan Lin received BS and $\mathrm{PhD}$ degrees in the Department of Light Sources and Illuminating Engineering, Fudan University in 1999 and 2005, respectively. She is now a full professor at the Department of Light Sources and Illuminating Engineering, Fudan University. Her research interests include VC and color vision. She has published more than 100 academic articles. She is the chair of CIE TC 1-91 and is active in the field of color and vision. 